\title{
Silk Fibroin-based Scaffolds with Controlled Delivery Order of VEGF and BDNF for Cavernous Nerve Regeneration
}

Yaopeng Zhang ${ }^{\dagger}{ }^{*}$ (Co-first author), Jianwen Huang ${ }^{\ddagger}$ (Co-first author), Li Huang ${ }^{\dagger}$, Qiangqiang $\mathrm{Liu}^{\dagger}$, Huili Shao ${ }^{\dagger}$, Xuechao $\mathrm{Hu}^{\dagger}$, Lujie Song ${ }^{\ddagger} * *$

${ }^{\dagger}$ State Key Laboratory for Modification of Chemical Fibers and Polymer Materials, College of Materials Science and Engineering, Donghua University, Shanghai 201620, P. R. China

†Department of Urology, Shanghai Jiao Tong University Affiliated Sixth People's Hospital, Shanghai 200233, P. R. China

zyp@dhu.edu.cn; $\quad 309018008 @ q q . c o m ; \quad$ huangli2015xy@ 163.com; liuqiangqiang90@163.com; $\quad$ hlshao@dhu.edu.cn; $\quad$ xchu@dhu.edu.cn; ljsong@sjtu.edu.cn 


\section{Porosity Calculation}

The porosity $\varepsilon$ of RSF scaffold was calculated according to the following equation:

$$
\varepsilon=\left(1-\frac{\rho}{\rho_{0}}\right) \times 100
$$

The bulk densities $\rho\left(\mathrm{g} / \mathrm{cm}^{3}\right)$ of the electrospun RSF meshes were determined using the weight of samples with defined area and thickness. The polymer density $\rho_{0}$ of RSF was measured with Ultrapycnometer 1000, Quantachrome. The test was performed with $10 \mathrm{~mL}$ sample cell, $0.15 \mathrm{MPa}$ nitrogen purging at $25{ }^{\circ} \mathrm{C}$.

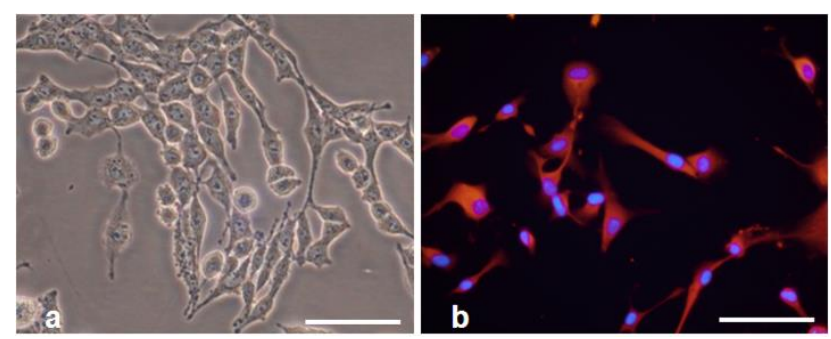

Figure S1 Morphology and identification of SCs before seeding on scaffolds. (a) Optical image of SCs on TCPS and (b) immunofluorescence staining of SCs on coverslip using monoclonal antibodies (anti-S100 $\beta$ protein). The length of scale bars are $20 \mu \mathrm{m}$. 